\title{
ASPECTOS MORFOANATÔMICOS E FISIOLÓGICOS DE Swietenia macrophylla KING SUBMETIDA A DUAS CONDIÇÕES DE INUNDAÇÃO
}

Manoel Euclides do Nascimento ${ }^{1}$, Raimundo Lázaro Moraes da Cunha ${ }^{1}$, Jessivaldo Rodrigues Galvão ${ }^{1}$, Pedro Paulo da Costa Alves Filho ${ }^{1}$, Mira Ketlen Carvalho Nascimento ${ }^{1}$, Rafaela Furtado da Cunha ${ }^{1}$, Antônio Vinicius Corrêa Barbosa ${ }^{1}$

${ }^{1}$ Universidade Federal Rural da Amazônia, E-mail: manoelnascimento@ufra.edu.br, azaro.cunha@ufra.edu.br, jessivaldo.galvao@ufra.edu.br, pp.alvesfilho@yahoo.com.br, pp.alvesfilho@yahoo.com.br, mira.ketlen@yahoo.com.br, rafaelacunha_agro@yahoo.com.br, vinicius.barbosa@ufra.edu.br

\section{RESUMO}

Visando a elucidar aspectos da tolerância ao alagamento, plantas de Swietenia macrophylla King foram submetidas a duas condições de inundação: solo inundado continuamente e solo com inundação intermitente por 72 horas. A sobrevivência, o crescimento, a variação anatômica foliar, a condutância estomática e a transpiração foram avaliadas. Houve mortalidade de $30 \%$ das plantas inundadas continuamente. Dentre as $70 \%$ sobreviventes, o crescimento do caule, a área foliar, o número de folhas e os parâmetros anatômicos avaliados, foram inferiores aos das plantas cultivadas em solo com inundação intermitente; massa seca da raiz e da parte aérea, teor de clorofila, relação dos diâmetros polar e equatorial dos estômatos e espessura do mesofilo foram semelhantes nas plantas cultivadas nos dois ambientes estudados, mas foram inferiores aos apresentados pelas plantas cultivadas em solo drenado. A inundação provocou redução na condutância estomática e na transpiração. Sugere-se que S. macrophylla possui intolerância a inundação do solo.

Palavras-chave: Tolerância à inundação, hipoxia, condutância estomática, transpiração

\section{MORPHOANATOMIC AND PHYSIOLOGICAL ASPECTS OF Swietenia macrophylla KING SUBMITTED TO TWO FLOOD CONDITIONS}

\begin{abstract}
To elucidate aspects of flooding tolerance, plants of Swietenia macrophylla King were subjected to two flood conditions: continuously flooded soil and soil with intermittent flooding for 72 hours. The survival, growth, leaf anatomical variation, stomatal conductance and transpiration were evaluated. The mortality rate of the continuously flooded plants was $30 \%$. Among the $70 \%$ survivors, the growth of the stem, leaf area, leaf number and anatomical parameters assessed were lower than those of plants grown in soil with intermittent flooding; dry
\end{abstract}


mass of the root and the shoot, chlorophyll content, ratio of polar and equatorial diameters of stomata and mesophyll thickness were similar in plants grown in both environments studied, but were lower than those presented by the plants grown in drained soil. The flooding caused a reduction in stomatal conductance and transpiration. It is suggested that S. macrophylla is intolerant to soil flooding.

Keywords: Tolerance to flooding, hypoxia, stomatal conductance, transpiration

\section{INTRODUÇ̃̃O}

A recuperação e ou a restauração de áreas degradadas na Amazônia, normalmente ocorre através do plantio de espécies nativas. Porém, um dos fatores limitante para o estabelecimento dessas espécies são as condições adequadas de disponibilidade hídrica.

A presença de vastas áreas alagáveis na Amazônia resulta da combinação de relevos baixos e de altas precipitações, características da região. Embora elevada, a precipitação não se distribui de maneira uniforme, mas aumenta ao longo de um gradiente de sudeste para noroeste da bacia amazônica (SIOLI, 1991). A sazonalidade do padrão de precipitação define ao longo do ano uma estação seca e uma estação chuvosa (SALATI \& VOSE, 1984). Como os grandes rios recebem o somatório da precipitação de toda a bacia de drenagem, as diferenças sazonais e geográficas na distribuição das chuvas levam a flutuações do nível da água desses rios, determinando, anualmente, a existência de uma fase de águas altas, a fase aquática, alternada com uma fase de águas baixas, a fase terrestre (JUNKET al., 1989). A diferença média entre o pico da fase aquática e o pico da fase terrestre pode atingir mais de 10 metros.

Swietenia macrophylla King é uma espécie arbórea que ocorre naturalmente na Amazônia Brasileira e, devido ao seu potencial econômico e ecológico tem sido apontada como espécie promissora para recuperação de áreas alteradas ou degradadas, especialmente aquelas de preservação permanente.

Em seu habitat natural, é de grande porte podendo atingir de 40 a $60 \mathrm{~m}$ de altura e diâmetro de $210 \mathrm{~cm}(1,30 \mathrm{~m}$ acima do solo). É planta heliófila, sendo tolerante à sombra durante a fase jovem. É caducifólia quando ocorre em regiões áridas. Ocorre de forma dispersa em florestas higrófilas subcaducifólias de baixas altitudes com precipitação média anual de 1600 a 2500 
mm e curto período seco de 4 meses. A temperatura na área de ocorrência natural oscila entre 24 e $27^{\circ} \mathrm{C}$ (média do mês mais frio em torno de $18^{\circ} \mathrm{C}$ ). Nas zonas de floresta higrófila perenifólia, prefere solos com reduzida capacidade de retenção de água. Suporta inundações durante o período de chuvas. No entanto, não tolera longos períodos de solo seco.

No decorrer do processo evolutivo, espécies tolerantes desenvolveram várias estratégias que as capacitaram a ocupar áreas sujeitas a inundação do solo. Dentre estas estratégias, ocorrem alterações morfoanatômicas que auxiliam a aeração interna da planta e alterações metabólicas, com diminuição no consumo de energia, ativação de rotas anaeróbias e menor investimento no crescimento (MEDRI et al., 2002; ALMEIDA; VALLE, 2007).

Algumas respostas das plantas a inundação podem incluir senescência foliar, redução no crescimento de caules e raízes, desenvolvimento de raízes adventícias, formação de aerênquima, hipertrofia de lenticelas e alterações nos pigmentos foliares e na Rabisco (PEZESHKI, 2001; MIELKE et al., 2003; EVANS, 2004; POVH et al., 2005; COSTA et al., 2006; MANO et al., 2006; HERRERA et al., 2008; HENRIQUE et al., 2010; SHIMAMURA et al., 2010).
Limitações estomáticas e não estomáticas são responsáveis pelo decréscimo na fotossíntese (KOSLOWSKI, 1997; MEDRI et al., 2007; PARENT et al., 2008). Apesar das condições desfavoráveis, muitos estudos mostram que o alagamento não impede completamente a ocorrência de determinados processos fisiológicos.

O estudo das características adaptativas de espécies tropicais é importante para elucidar alguns aspectos da sobrevivência em solo inundado. Este experimento foi conduzido com objetivo de conhecer as variações na transpiração, condutância estomática, crescimento e desenvolvimento e possíveis alterações morfoanatômicas de plantas jovens de mogno cultivadas em solo sob duas condições de inundação.

\section{MATERIAL E MÉTODOS}

O experimento foi conduzido em casa de vegetação da Universidade Federal Rural da Amazônia, em Belém, PA $\left(01^{\circ} 27^{\prime} 21^{\prime}, \quad \mathrm{S}\right.$ e 48³0'16', W). A semeadura foi realizada em bandejas e, após 20 dias, selecionou-se as plantas pela sua uniformidade e aspecto nutricional, transferidas para vasos plásticos (20L), mantendo-se uma planta após desbaste. Os vasos foram preenchidos com solo arenoso e 
esterco de curral (3:1). Em seguida, as plantas foram transferidas para o local do experimento onde passaram por um período de aclimatação de 45 dias, quando foram submetidas por 30 dias a três tratamentos: solo irrigado próximo capacidade de campo (IC), que serviu como controle; solo com inundação intermitente por 72 horas (AI) e solo com inundação contínua (AC). Por ocasião da imposição dos tratamentos as plantas apresentaram, em média, $25 \mathrm{~cm}$ de altura e seis folhas maduras. Durante o período do experimento, os valores médios da temperatura ambiente, umidade relativa do ar eradiação fotossinteticamente ativa foram, respectivamente, $29,3{ }^{\circ} \mathrm{C}, 70 \%$ e 495 $\mu \mathrm{mol} \mathrm{m} \mathrm{m}^{-2}$.

A inundação foi obtida colocando-se o vaso com a planta, dentro de outro vaso maior que recebeu água, mantendo-se o nível a cinco centímetros acima do solo. A cada72 horas, os vasos com as plantas submetidas à inundação intermitente eram retirados dos vasos maiores e deixados drenando pelo mesmo período de tempo, quando eram submetidos novamente a inundação, até completar 30 dias. A condutância estomática e a transpiração foram determinadas, exceto a primeira medição, a intervalos de três dias, por um porômetro portátil de equilíbrio dinâmico (modelo Li 1600, LiCor, Nebraska, USA), sob luz e $\mathrm{CO}_{2}$ ambiente, entre 9 e 10 horas da manhã, em folíolos totalmente expandidos do segundo nó a partir do ápice de cada planta. Decorridos os 30 dias de inundação, foi determinada a área foliar, através do programa Image J; altura do caule e comprimento de raiz com régua milimétrica; diâmetro do caule a seis centímetros acima do coleto com o uso de paquímetro, teor de clorofila com SPAD-502 da Minolta e a contagem do número de folhas. Também foi contado o número de plantas mortas durante o período experimental. Para a determinação da biomassa seca, as plantas foram separadas em raiz, e parte aérea e colocadas para secar em estufa a $60{ }^{\circ} \mathrm{C}$ por quatro dias, tempo suficiente para atingir peso constante e, pesadas embalança semianalítica. As observações anatômicas foram obtidas em nível foliar, onde os parâmetros frequência e desenvolvimento estomático, e espessura do mesofilo foram registrados a partir de material fixado em FAA por 72 horas e posteriormente transferido para o álcool 70 \% aquoso. A confecção das lâminas semipermanentes seguiu o método usual em anatomia vegetal, duplamente coradas com azul de astra e safranina e montadas em glicerina $75 \%$ aquosa. As contagens de 
estômato foram realizadas em $0,017 \mathrm{~mm}^{2} \mathrm{de}$ área foliar, as medições a partir de fotos padronizadas foram obtidas com auxílio do programa Image tool 3.0. O delineamento experimental foi o de blocos ao acaso, com seis repetições com uma planta por cada unidade experimental. Os dados foram submetidos à análise de variância e as médias comparadas pelo teste de Tukey ao nível de 5\% de probabilidade.

\section{RESULTADOS E DISCUSSÃO}

A inundação causou redução na condutância estomática $(g s)$ em todos os períodos avaliados (Figura 1A). Entretanto, em solo com inundação intermitente (AI) a redução foi gradual, atingindo $25 \%$ aos seis dias, $60 \%$ aos 12 dias e $70 \%$ nos demais dia, em relação às plantas controle (IC). Já, na inundação contínua (AC), a gsreduziu bruscamente em 70\%, logo no sexto dia; não havendo diferença significativa entre as avaliações nos demais dias. A transpiração (E) também diminuiu nas plantas inundadas (Figura 1B). À semelhança da gs, ocorreu diminuição gradual de $E$ nas plantas sob inundação intermitente. Porém, a redução $\operatorname{de} E$ foi mais expressiva, com cerca de $40 \%$ no sexto dia.

A menor capacidade da raiz em absorver água tem sido a principal causa do fechamento parcial ou total dos estômatos (KREUZWIESER et al., 2004; PIERCE et al., 2010), visto que baixa produção de ATP na respiração anaeróbica de raizes hipóxicas pode limitar a energia disponivel aos processos celulares, acarretando redução ou morte das raizes (BARRETT-LENNARD, 2003). A redução na condutância estomática em plantas inundadas sugere que o fechamento estomático causa redução na taxa fotossintética (MIELKE et al., 2003). De fato, a Tabela 1mostra que $S$. Macrophylla submetida à inundação por 30 dias apresentou acentuada redução tanto da massa de raízes quanto da massa aérea, sobretudo em solo com inundaçãocontinua.

Do total $30 \%$ das plantas não sobreviveram à inundação contínua do solo (Tabela 1).

A partir do décimo dia de alagamento, constatou-se o aparecimento de clorose, senescência foliar e formação de lenticelas hipertróficas. A altura do caule, a área foliar e o número de folhas apresentaram valores significativamente distintos entre solo com inundação intermitente por 72 horas e solo com inundação contínua (Tabela 1). Tais resultados mostram que a inundação contínua foi mais severa, apresentando redução em cerca de 50\% nesses parâmetros avaliados. 


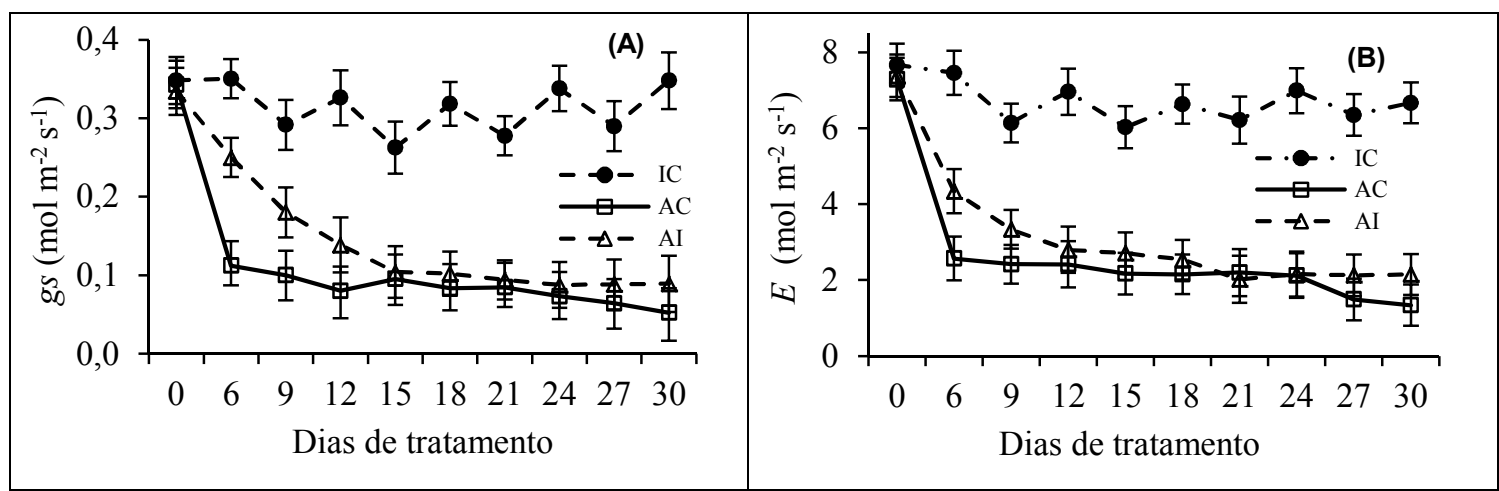

Figura 1. Condutância estomática $(g s)$ - A e transpiração $(E)$ - B em folhas de plantas jovens de Swietenia macrophylla, submetidas a três condições hídricas: irrigado na capacidade de campo (IC), alagamento intermitente por 72 horas (AI) e alagamento contínuo (AC).

Tabela 1. Mortalidade, órgãos vegetativos e teor de clorofila de plantas jovens de Swietenia macrophylla, submetidas a três condições hídricas: irrigado na capacidade de campo (IC); alagamento intermitente por 72 horas (AI) e alagamento contínuo (AC).

\begin{tabular}{lllllllll}
\hline Tratamentos & M (\%) & AC (cm) & AF (cm2) & NF (un) & CR (cm) & MSA(g) & MSR (g) & TC \\
\hline IC & 0a & $78,87 \mathrm{a}$ & $492,99 \mathrm{a}$ & $19,62 \mathrm{a}$ & $43,30 \mathrm{a}$ & $1,64 \mathrm{a}$ & $1,38 \mathrm{a}$ & $66,25 \mathrm{a}$ \\
& & & & & & & & \\
$\mathrm{AI}$ & $0 \mathrm{a}$ & $49,67 \mathrm{~b}$ & $396,73 \mathrm{~b}$ & $10,75 \mathrm{~b}$ & $28,40 \mathrm{~b}$ & $1,12 \mathrm{~b}$ & $0,88 \mathrm{~b}$ & $55,50 \mathrm{~b}$ \\
$\mathrm{AC}$ & $30 \mathrm{~b}$ & $33,50 \mathrm{c}$ & $257,33 \mathrm{c}$ & $7,75 \mathrm{c}$ & $29,13 \mathrm{~b}$ & $0,98 \mathrm{~b}$ & $0,70 \mathrm{~b}$ & $47,25 \mathrm{~b}$ \\
\hline $\mathrm{CV}(\%)$ & 14,53 & 14,13 & 19,11 & 11,57 & 13,54 & 27,21 & 23,49 & 11,53
\end{tabular}

Médias seguidas pelas mesmas letras nas colunas não diferem entre si pelo teste de Tukey a 5\% de probabilidade. Mortalidade (M), altura do caule (AC), área foliar (AF), número de folhas (NF), comprimento da raiz (CR), massa seca da parte aérea (MSA) e da raiz (MSR) e teor de clorofila (TC).

Já o comprimento da raiz, massa seca aérea, massa seca da raiz e teor de clorofila, apesar de não apresentarem diferenças significativas entre os solos inundados, foram reduzidos em cerca de $40 \%$, quando comparados ao tratamento testemunha.

Os resultados encontrados mostram um forte efeito da inundação do solo no crescimento e desenvolvimento de $S$. macrophylla. Os mesmos efeitos também foram apresentados por outras espécies arbóreas neotropicais, como em Lithraea molleoides (MEDRI et al., 2007), Cecropia pachystachya (BATISTA et al., 2008) e em Aegiphila sellowiana (MEDRI et al., 2012).No entanto, a maioria das espécies tolerantes neotropicais tem apresentado significativa redução do crescimento da planta como um todo, como foi observado por Mielkeet al. (2003) em Genipa americana. $\mathrm{O}$ efeito da inundação foi bastante severo em folhas de S. macrophylla, 
principalmente por ter o estresse estimulado a abscisão, sobretudo no solo com inundação contínua. A produção de novas folhas foi menor nas plantas inundadas, não sendo suficiente para compensar a perda de massa pela maior abscisão. Martinez et al. (2011) registraram decréscimo da área foliar em plantas de Hura crepitans cultivadas em solo inundado, como consequência da maior abscisão de folhas e menor produção de folhas novas. Medriet al. (2012), pelos mesmos motivos, verificaram que a hipoxia levou a uma menor área foliar total em Aegiphila sellowiana. Segundo Drew (1991), a inundação reduz a absorção e o transporte de íons, resultando em um estresse mineral para a parte aérea, geralmente, acompanhado de senescência e abscisão prematura de folhas.

A massa seca é importante parâmetro para avaliar a capacidade das espécies em tolerar a inundação. A fitomassa aérea e da raiz de $S$. macrophylla em solo alagado e intermitente, ao final do experimento, diminuiu com a inundação (Tabela 1). Os resultados permitem inferir que a redução da área foliar, a abscisão e a pouca produção de folhas novas contribuíram para tal fato. A diminuição da fitomassa é considerada uma resposta típica das plantas à inundação (MARTINEZ et al., 2011; MEDRI et al.,
2012), como o ocorrido com as seguintes espécies submetidas à inundação: Lithraea molleoides (redução de 50\%), Genipa americana (redução de até 35,6\%) e Himatanthus sucuuba (redução de 58\%) (MEDRI et al., 2007).

As mudanças na assimilação do carbono podem ser atribuídas a limitações fotossintéticas não relacionadas aos estômatos (HERRERA et al., 2008), como alterações nos pigmentos foliares (ISHIDA et al. 2002; GONÇALVES et al., 2010). O teor de clorofila nas folhas das plantas submetidas à inundação contínua e intermitente foi inferior ao das plantas testemunha (Tabela 1). De modo geral, reduções dos teores foliares de pigmentos cloroplastídicos têm sido verificadas em grande número de espécies sob condição de alagamento (ISHIDA et al. 2002; MAIA; PIEDADE 2002; CHEN et al. 2005; GONÇALVES et al., 2010). Provavelmente, a redução nos teores de clorofilas nas plantas inundadas, de certa forma, deva estar relacionada com a diminuição na quantidade de $\mathrm{O}_{2}$ disponível para as plantas em ambientes inundados, ocasionando restrições no metabolismo, como por exemplo, redução das atividades da enzima redutase do nitrato, prejudicando a absorção e o transporte de íons, tendo como consequência, redução nos 
teores de nitrogênio na parte aérea da planta, que por sua vez está intimamente relacionado com os teores de clorofilas (DREW, 1999; NETTO et al., 2005).

As variáveis anatômicas foliares sofreram influência do AI e AC. Com exceção da densidade estomática e da relação DPE, todas as demais variáveis apresentaram diferenças significativas de acordo com o teste de Tukey, quando comparadas a testemunha (Tabela 2).

A espessura do mesofilo foi mais reduzida nas plantas submetidas ao alagamento contínuo (Figura 2E).

O diâmetro polar e o diâmetro equatorial dos estômatos foram reduzidos igualmente pelo tratamento $\mathrm{AC}$ em cerca de $10 \%$ (Figura 2F), quando comparados a testemunha (Figuras 2A e 2B).

Tabela 2. Superfície e área foliar de plantas jovens de Swietenia macrophylla, submetidas a três condições hídricas: irrigado na capacidade de campo (Testemunha) (IC); alagamento intermitente por 72 horas (AI); e alagamento contínuo (AC).

\section{Superfície e mesófilo foliar}

\section{DET $\quad$ DP $(\mu \mathrm{m}) \quad$ DE $(\mu \mathrm{m}) \quad$ DPE $(\mu \mathrm{m}) \quad \mathrm{EMF}(\mu \mathrm{m})$}

\begin{tabular}{cccccc}
\hline IC & $26,76 \mathrm{a}$ & $75,20 \mathrm{a}$ & $60,20 \mathrm{ba}$ & $1,24 \mathrm{a}$ & $618,02 \mathrm{a}$ \\
$\mathbf{A I}$ & $28,92 \mathrm{a}$ & $70,69 \mathrm{~b}$ & $57,40 \mathrm{cb}$ & $1,22 \mathrm{a}$ & $598,14 \mathrm{ba}$ \\
$\mathbf{A C}$ & $23,36 \mathrm{a}$ & $69,52 \mathrm{~b}$ & $56,16 \mathrm{c}$ & $1,23 \mathrm{a}$ & $586,66 \mathrm{~b}$ \\
\hline $\mathbf{C V}(\%)$ & 14,55 & 6,50 & 6,89 & 7,52 & 2,80
\end{tabular}

Médias seguidas pelas mesmas letras na coluna não diferem entre si pelo teste de Tukey a 5\% de probabilidade. Densidade estomática (DET), diâmetro polar do estômato (DP), diâmetro equatorial do estômato (DE), razão dos diâmetros polar/equatorial dos estômatos (DPE), espessura do mesofilo foliar (EMF) e biomassa seca da parte aérea (BSA) e da parte subterrânea (BSS). 

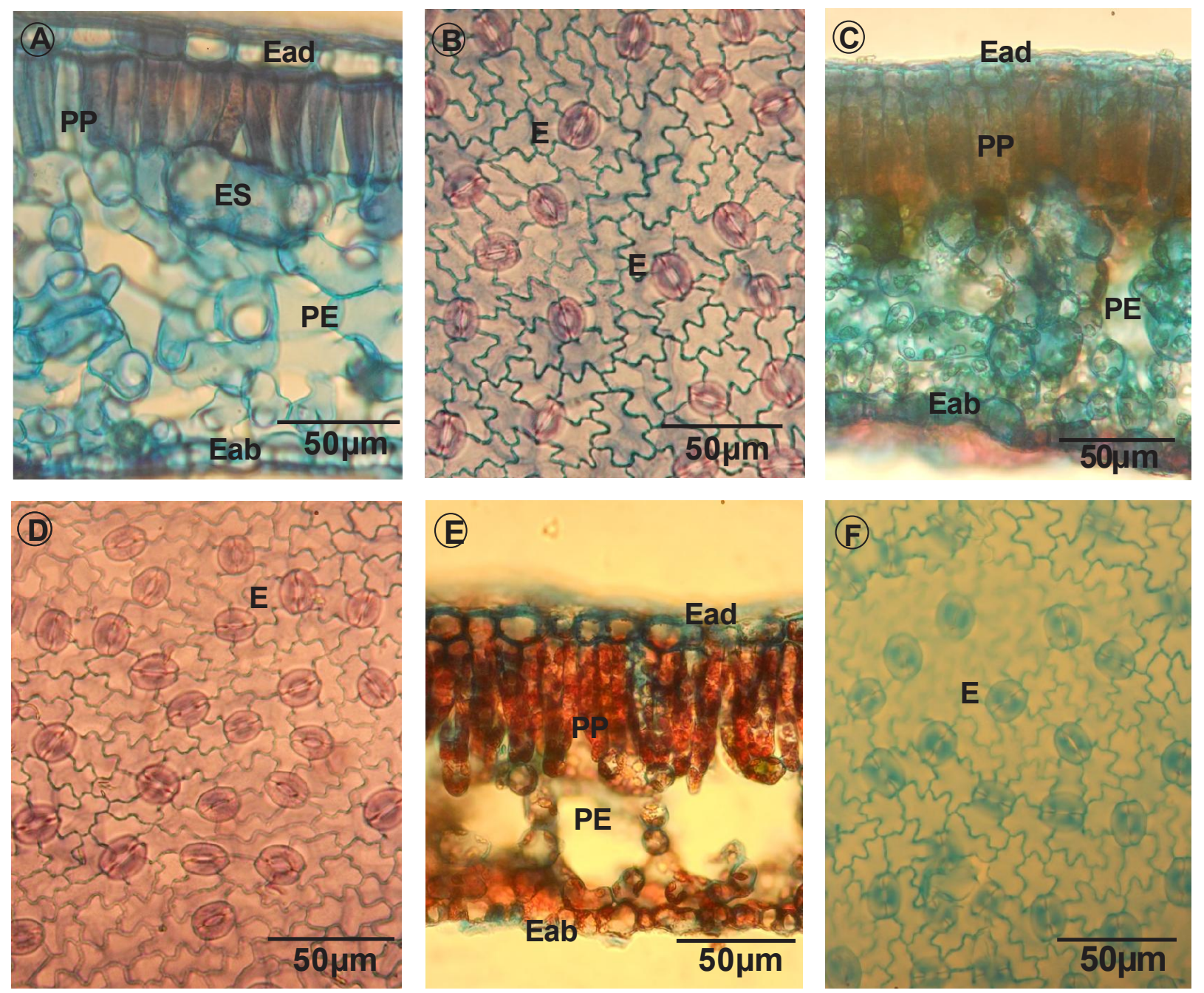

Figura 2. Superfície e mesofilo foliar submetidos a três regimes hídricos: A - mesofilo IC (irrigado na capacidade de campo); $\mathrm{B}$ - superfície foliar IC; $\mathrm{C}$ - mesofilo AI (alagado intermitente); D - superfície foliar AI; E - mesofilo AC (alagado constante) e F superfície foliar AC. Ead(face epidérmica adaxial), Eab (face epidérmica abaxial), PP (parênquima paliçádico), PE (parênquima esponjoso), ES (estrutura secretora) e $\mathbf{E}$ (estômato).

Os maiores índices obtidos na DPE revelam estômatos mais elipsoides, mais funcionalidade e maior eficiência fotossintética. Tais resultados ratificam os estudos de Martins et al., (2009); Rosato et al., (2009); Hogewoning et al., (2010), onde os estômatos com maior índice de razão entre o diâmetro polar e equatorial, também possuem menores diâmetros dos ostíolos, apresentam características dinâmicas, mais rapidez, portanto alta condutância estomática, maior eficiência fotossintética e uso eficiente da água (DRAKE; FROEND e FRANKS, 2013; FRANKS; DRAKE e 
BEERLING, 2009). Estômatos com menor diâmetro do poro estomático estão relacionados a menor translocação de fotoassimilados, comprovados pela menor espessura do mesofilo nos tratamentos AI e AC (Figuras 2D e 2F), refletindo no crescimento caulinar, no número de folhas e área foliar, corroborado pelo crescimento da raiz e espessura do coleto (MARTINS et al., 2009).

Embora, 70\% das plantas em inundação contínua e 100\% das plantas em inundação intermitente tenham sobrevivido, apresentaram desenvolvimento limitado, com redução da área foliar, abscisão de folhas, redução no teor de clorofila e queda na condutância estomática e transpiração, corroborados pela diminuição da frequência estomática, do diâmetro polar e equatorial dos estômatos, e da redução da espessura do mesofilo da área foliar e do número de folhas.

\section{CONCLUSÃO}

A inundação contínua ou intermitente por 72 horas, durante 30 dias, implicou redução no desenvolvimento das plantas jovem de Swietenia macrophylla, com consequente redução da atividade fotossintética. Tais características sugerem que $S$. macrophylla é intolerante ao estresse causado por essas variações ambientais.

\section{REFERÊNCIAS BIBLIOGRÁFICAS}

ALMEIDA, A. A. F.; VALLE, R. R. 2007. Ecophysiology of the cacao tree. Brazilian Journal Plant Physiology, Campinas, v. 19, p. 425-448, ARMSTRONG, W.; BRAENDLE, R. JACKSON, M.B. 1994. Mechanisms of flood tolerance in plants. Acta Botanica Neerlandica, Oxford. v. 43, p. 307-358.

BARRETT-LENNARD, E.G. 2003. The interaction between waterlogging and salinity in higher plants: causes, consequences and implications. Plant and Soil, Perth. p.35-54.

BATISTA, C.U.N.; MEDRI, M.E.; BIANCHINI, E.; MEDRI, C.; PIMENTA, J.A. 2008. Tolerância à inundação de Cecropia pachystachya Trec. (Cecropiaceae): aspectos ecofisiológicos e morfoanatômicos. Acta Botanica Brasilica, Feira de Santana, v. 22, n. 1, p. 91-98.

CHEN, H.; QUALLS, R.G.; BLANK, R.R. 2005. Effect of soil flooding on photosynthesis, carbohydrate partitioning and uptake in the invasive exotic Lepidium latifolium. Aquatic Botany, Amsterdam. 82: 250-268.

COSTA, A.M.; GOBBI, E.L.; DEMUNER, V.G.; HEBLING, S.A.O. 2006. Efeito da inundação do solo sobre o crescimento inicial de Schizolobium parahyba (Vell.) S.F. Blake, guapuruvu. Natureza online, Santa Tereza, v. 4, n. 1, p. 7-13. 
DRAKE， P.L.; FROEND， R.H.; FRANKS, P.J.2013. Smaller, faster stomata: scaling of stomatal size, rate of response, and stomatal conductance. Journal of Experimental Botany. Oxford. v. 64, n. 2, p. 495-505.

DREW, M. C. 1991. Oxygen deficiency in the root environment and plant mineral nutrition. In: JACKSON, M. B.; LAMBERS, D. D. (Ed.). Plant life under deprivation. Michigan The Hague: SPB Academic Publishing, p. 303-316.

EVANS, D.E. 2004. Aerenchyma formation. New Phytologist. Oxford. 161: 35-49.

FRANKS， P.J.; DRAKE， P.L.; BEERLING, D.J. 2009. Plasticity in maximum stomatal conductance constrained by negative correlation between stomatal size and density: an analysis using Eucalyptus globulus. Plant, Celland \& Environment, Oxford. v. 32, p.1737-1748.

GONÇALVES, J.F.C.; SILVA, C.E.M.; GUIMARÃES, D.G.; BERNARDES, R.S. 2010. Análise dos transientes da fluorescência da clorofila a de plantas jovens de Carapa guianensis e de Dipteryx odorata submetidas a dois ambientes de luz. Acta amazônica, Manaus. (1): 89-98.

HENRIQUE, P. C.; ALVES, J. D.; GOULART P. F. P.; DEUNER. S.; SILVEIRA, N. M.; ZANANDREA. I.; CASTRO. E. M. 2010. Características fisiológicas e anatômicas de plantas de sibipiruna submetidas à hipóxia. Ciência Rural, Santa Maria - RS, v. 40, n. 1.

HERRERA, A.; TEZARA, W.; MARÍN, O.; RENGIFO, E. 2008. Stomatal and non-stomatal limitations of photosynthesis in trees of a tropical seasonally flooded forest. Physiologia Plantarum, Lund, v. 134, n. 1, p. 4148.

HOGEWONING, S. W.; TROUWBORST, G.; MALJAARS, POORTER, H.; VAN IEPEREN,W.; HARBINSON, J. 2010. Blue light dose-responses of leaf photosynthesis, morphology, and chemical composition of Cucumis sativus grown under different combinations of red and blue light. Journal of Experimental Botany, v. 61, n. 11, p. 3107-3117.

ISHIDA, F. Y.; OLIVEIRA, L. E. M.; CARVALHO, C. J. R.; ALVES, J. D. 2002. Efeito da inundação parcial e total sobre o crescimento, teor de clorofila e fluorescência de Setaria anceps e Paspalum repens. Ciência e Agrotecnologia. Lavras. 26(6): 11521159.

KREUZWIESER, J.;

PAPADOPOULOU, E.; RENNENBERG, H. 2004. Interaction of flooding with carbon metabolism of forest trees. Plant Biol, (Stuttg), v. 6, n. 3, p. 299-306.

MAIA, L. A.; PIEDADE, M. T. 2002. Influência do pulso de inundação na fenologia foliar e conteúdo de clorofila em duas espécies da floresta de igapó na Amazônia Central, Brasil. Acta amazônica, Manaus. 32(1): 5564.

MANO, Y.; OMORI, F.; TAKAMIZO, T.; KINDIGER, B.; McK. BIRD, R.; LOAISIGA, C.H. 2006. Variation for root aerenchyma formation in flooded and non-flooded maize and teosinte 
seedlings. Plant and Soil, Perth. 281: 269-279.

MARTINEZ, G. B.; MOURÃO, M; BRIENZA JUNIOR, S. 2011. Respostas morfofisiológicas de plantas de açacu (Hura crepitans L.) provenientes de várzeas do rio amazonas: efeito da anoxia do solo, Revista Árvore, Viçosa-MG, v.35, n.6, p.1155-1164.

MARTINS, J. R.; ALVARENGA, A. A.; CASTRO, E. M. DE; DA SILVA, A. P. O.; OLIVEIRA, C.; ALVES, E. 2009. Anatomia foliar de plantas de alfavaca-cravo cultivadas sob malhas coloridas. Ciência Rural, Santa Maria. v.39, n.1, p.82-87.

MEDRI, M. E.; BIANCHINI, E.; PIMENTA, J. A.; COLLI, S.; MULLER, C. Estudos sobre a tolerância ao alagamento em espécies arbóreas nativas da bacia do rio Tibagi. In: MEDRI, M. E.; BIANCHINI, E.; SHIBATTA, O. A.; PIMENTA, J. A. 2002. (Ed.). A bacia do Rio Tibagi. Londrina: Edição dos editores, v. 1, p. 133-172.

MEDRI, M. E.; FERREIRA, A. C.; KOLB, R. M.; BIANCHINI, E.; PIMENTA, J. A.; DAVANSOFABRO, V. M.; MEDRI, C. 2007. Alterações morfoanatômicas em plantas de Lithraea molleoides (Vell.) Engl. submetidas ao alagamento. Acta Scientiarum Biological Sciences, Maringá, v. 29, n. 1, p. 15-22.

MIELKE, M.S.; ALMEIDA, A.F.; GOMES, F.B.; AGUILAR, M.A.G.; MANGABEIRA, P.A.O. 2003. Leaf gas exchange, chlorophyll fluorescence and growth responses of Genipa americana seedlings to soil flooding. Environmental and
Experimental Botany, Oxford. v. 50, p. 221-231.

NETTO, A. T.; CAMPOSTRINI, E.; OLIVEIRA, J.G.; BRESSAN-SMITH, R.E. 2005. Photosynthetic pigments, nitrogen, chlorophyll a fluorescence and SPAD-502 readings in coffee leaves. Scientia Horticulturae 104: 199-209.

PARENT C. CAPELLI, N. BERGER, A. CRÈVECOEUR, M. DAT, J. F. 2008. An Overview of Plant Responses to Soil Waterlogging. Plant Stress, Israel. v. 2, n. 1, p.2027.

PIERCE, S. C.; MOORE, M. T.; LARSEN, D.; PEZESHKI, S. R. 2010. Macronutrient (N, P, K) and Redoximorphic Metal (Fe, Mn) Allocation in Leersia oryzoides (Rice Cutgrass) Grown Under Different Flood Regimes. Water Air Soil Pollut, Canadá. v. 207, p. 73-84.

POVH, J. A.; RUBINFILHO, C. J.; MOURÃO, K. S. M.; PINTO, D. D. 2005. Respostas morfológicas e anatômicas de plantas jovens de Chorisia speciosa A. St.Hil. (Bombacaceae) sob condições de alagamento. Acta Scientiarum Biological Sciences, Maringá. v.27, n.3, p.195-202.

ROSSATTO, D. R.; HOFFMANN, W. A.; FRANCO, A. C. 2009. Características estomáticas de pares congenéricos de cerrado e mata de galeria crescendo numa região transicional no Brasil central. Acta Botânica Brasilica, Belo Horizonte. v. 23 , n. 2.

SALATI, E.; VOSE, P. B. 1984. Amazon basin: a system in equilibrium. Science, v. 225, p. 129-138. 
SIOLI, H. 1991. Amazônia. Fundamentos da Ecologia da Maior Região de Florestas Tropicais. Petrópolis, Rio de Janeiro Brasil, 72 pp.: Editora Vozes.

SHIMAMURA, S.; YAMAMOTO, R.; NAKAMURA, T.; SHIMADA, S.; KOMATSU, $\quad$ S. $\quad$ S. 2010. Hypertrophiclenticelsandsecondaryaer enchymaenableoxygentransportto roots of soybean in flooded soil. Annals of Botany, Oxford. v. 106, p. 277-284. 